\title{
FATORES PROGNÓSTICOS NA RESSECÇÃO DE METÁSTASES HEPÁTICAS DE CÂNCER COLORRETAL
}

\author{
Aljamir Duarte CHEDID ${ }^{1,2}$, Maitê de Mello VILLWOCK ${ }^{2}$, Marcio Fernandes CHEDID $^{2}$ e Luiz ROHDE ${ }^{3}$
}

\begin{abstract}
RESUMO - Objetivo - Determinar o impacto de fatores prognósticos na sobrevida de pacientes com metástases hepáticas ressecadas e originadas de câncer colorretal. Casuística e Métodos - Foram analisados os prontuários de 28 pacientes submetidos a ressecção hepática de metástases de câncer colorretal de abril de 1992 a setembro de 2001. Foram realizadas 38 ressecções (8 pacientes com mais de uma ressecção no mesmo tempo cirúrgico e 2 pacientes submetidos a re-ressecções). Todos haviam sido submetidos previamente a ressecção do tumor primário. Utilizou-se protocolo de rastreamento de metástases hepáticas que incluiu revisões clínicas trimestrais, ecografia abdominal e dosagem de CEA até se completarem 5 anos de seguimento e após, semestralmente. Os fatores prognósticos estudados foram: estágio do tumor primário, tamanho das metástases $>5 \mathrm{~cm}$, intervalo entre ressecção do tumor primário e surgimento da metástase $<1$ ano, CEA $>100$ $\mathrm{ng} / \mathrm{mL}$, margens cirúrgicas $<1 \mathrm{~cm}$ e doença metastática extra-hepática. O estudo foi retrospectivo e a análise estatística foi feita pela curva de Kaplan-Meier, log-rank e regressão de Cox. Resultados - A morbidade foi 39,3\% e a mortalidade operatória foi 3,6\%. A sobrevida em 5 anos foi de $35 \%$. Os fatores prognósticos independentes adversos foram: intervalo $<1$ ano entre ressecção do tumor primário e surgimento da metástase, e doença metastática extra-hepática. Conclusões - A ressecção hepática de metástases de câncer colorretal é um procedimento seguro com sobrevida em 5 anos acima dos $30 \%$. Foram fatores prognósticos independentes adversos: doença metastática extra-hepática e intervalo $<1$ ano entre ressecção do tumor primário e surgimento da metástase.
\end{abstract}

DESCRITORES - Neoplasias colorretais. Neoplasias hepáticas. Metástase neoplásica. Prognóstico.

\section{INTRODUÇÃO}

Aproximadamente 150.000 novos casos de câncer colorretal são tratados anualmente nos Estados Unidos e cerca da metade desses pacientes terão metástases hepáticas no curso de sua doença $^{(39)}$. No Brasil, segundo dados do Instituto Nacional do Câncer ${ }^{(6)}$, a incidência aproximada para o ano 2001 era de 20.000 casos. A sobrevida de pacientes com metástases hepáticas de câncer colorretal não-ressecadas é em torno de $9 \operatorname{meses}^{(29)}$. As metástases hepáticas de câncer colorretal se desenvolvem em geral nos 2 anos subseqüentes à ressecção do tumor primário, são mais freqüentes nos pacientes com estágio Dukes $C^{(32)}$ e são sincrônicas com o tumor primário em torno de $25 \%$ das vezes ${ }^{(4)}$.

Em 1963, WOODINTON e WAUGH ${ }^{(44)}$ relataram sobrevida de $20 \%$ em 5 anos em pacientes submetidos a ressecção hepática de metástases de câncer colorretal.

Em 1976, WILSON e ADSON ${ }^{(43)}$ relataram sobrevida de $28 \%$ em 5 anos e recomendaram tratamento agressivo para lesões aparentemente solitárias.
Em 1977, FOSTER e BERMANN ${ }^{(15)}$, analisando criticamente o estágio da cirurgia hepática, fortaleceram a utilização da abordagem cirúrgica para tumores sólidos do fígado.

Atualmente, com uma seleção adequada dos pacientes, a ressecção hepática é o tratamento de escolha para metástases de câncer colorretal confinadas ao fígado e resulta em sobrevida que varia de $25 \%$ a $40 \%$ em várias séries ${ }^{(1,13,19,20,34}$, 37). Ocasionalmente, a ressecção total de doença metastática extra-hepática intra-abdominal combinada com a ressecção hepática e mesmo a ressecção de metástases pulmonares, tem se justificado com aumento da sobrevida ${ }^{(20,25)}$. Os pacientes com metástases hepáticas de câncer colorretal representam um grupo de comportamento biológico exclusivo visto que outros tumores do trato gastrointestinal, quando metastáticos no fígado, não apresentam padrões de resposta favorável com a ressecção ${ }^{(7)}$. Vários estudos têm sido realizados na tentativa de definir fatores prognósticos quanto à recidiva dessas lesões no fígado, na sobrevida e eventual cura dos pacientes $\operatorname{operados}^{(1,13,19,20,24,29,31,34,37,39)}$. A análise de fatores

${ }^{1}$ Serviço de Cirurgia do Hospital Ernesto Dornelles; ${ }^{2}$ Serviço de Transplante Hepático Adulto do Hospital de Clínicas de Porto Alegre e ${ }^{3}$ Departamento de Cirurgia da Faculdade de Medicina da Universidade Federal do Rio Grande do Sul, Porto Alegre, RS.

Endereço para correspondência: Dr. Aljamir Duarte Chedid - Rua Marechal Andréa 300/201 - Bairro Boa Vista - $91340-400$ - Porto Alegre, RS. E-mail: marcioch@terra.com.br 
prognósticos pode ser útil para selecionar pacientes para a ressecção hepática, delinear grupos de risco, estimar o tempo de sobrevida e oferecer aos pacientes dados objetivos sobre o seu prognóstico ${ }^{(39)}$.

No Brasil há poucas publicações com estudo estatístico de fatores prognósticos de metástases hepáticas de câncer colorretal ${ }^{(11)}$.

\section{CASUÍSTICA E MÉTODO}

Foram revisados, por um mesmo cirurgião (ADC), os prontuários de 34 pacientes submetidos a ressecção hepática de metástase de câncer colorretal nos hospitais Ernesto Dornelles e Moinhos de Vento, em Porto Alegre, RS, entre abril de 1992 e setembro de 2001. Todos os pacientes foram previamente submetidos a ressecção do tumor primário e a rotina de seguimento para rastreamento de metástases hepáticas incluiu revisões clínicas trimestrais com ecografia abdominal e dosagem de CEA até 5 anos de seguimento. Após 5 anos, as revisões passaram a ser semestrais. A seleção de pacientes para a ressecção hepática foi feita seguindo os seguintes critérios: a) ausência de sinais de doença tumoral disseminada avaliada por tomografia computadorizada do abdome, tórax e crânio; b) possibilidade de preservação de quantidade adequada de parênquima hepático após a ressecção, avaliada através de tomografia computadorizada de abdome e perfil laboratorial hepático; c) condições clínicas dos pacientes a serem submetidos a hepatectomia obtidas através de avaliação pré-operatória de rotina para cirurgias de grande porte e status de performance igual ou maior que 80 (índice de Karnofski). Em dois pacientes foi realizada arteriografia para definição diagnóstica. Da amostra inicial de 34 pacientes, foram excluídos 6 pacientes cujos prontuários não continham todas as informações requeridas no protocolo. Os dados obtidos foram sexo, idade, localização e estágio do tumor primário; nível de CEA do momento do diagnóstico da metástase hepática; características da metástase hepática: número, localização, momento do aparecimento e distribuição; detalhes cirúrgicos: tipo de ressecção, uso de transfusão sangüínea, tempo de internação, morbidade e mortalidade cirúrgica (óbitos ocorridos até o $30^{0}$ dia de pós-operatório); quimioterapia após a ressecção do tumor primário e sobrevida em 1, 2, 3, 4 e 5 anos. Foram estudados como fatores prognósticos: estágio Dukes $\mathrm{C}$ do tumor primário, tamanho da metástase maior que $5 \mathrm{~cm}$, intervalo menor que 1 ano entre ressecção do tumor primário e surgimento da metástase, nível de CEA maior que $100 \mathrm{ng} / \mathrm{mL}$, margens livres de tumor menores que $1 \mathrm{~cm}$ na metástase ressecada e presença de doença metastática extra-hepática no momento da cirurgia da metástase. Entre as duas nomenclaturas utilizadas para as ressecções hepáticas ${ }^{(9,10,17)}$, utilizou-se a de Couinaud ${ }^{(5,9,10)}$

$\mathrm{Na}$ análise estatística, foram utilizadas as curvas de sobrevida de Kaplan-Meier, log-rank para comparação da sobrevida, de acordo com o número de fatores prognósticos presentes, e a regressão de
Cox para controle dos fatores de confusão e interação ${ }^{(2)}$. Diferenças estatísticas foram consideradas significativas com $P<0,05$.

\section{RESULTADOS}

\section{Características da amostra estudada}

Do total de 28 pacientes operados, 13 eram do sexo masculino $(46,4 \%)$ e 15 do feminino (53,6\%). A média de idade foi de 60,14 anos.

O tumor primário estava localizado no intestino grosso em 18 pacientes $(64,2 \%)$ e no reto em 10 pacientes $(35,8 \%)$. Em 4 pacientes $(14,3 \%)$ tratava-se de adenocarcinoma bem diferenciado e em 24 $(85,7 \%)$ de adenocarcinoma moderadamente diferenciado. A metástase foi sincrônica com o tumor primário em 5 pacientes $(17,9 \%)$. Em 4 pacientes (14,3\%) foi diagnosticada durante o primeiro ano de seguimento e em 19 (67,9\%), após esse período.

Os níveis de CEA no momento do diagnóstico da metástase hepática variaram de 2 a $182 \mathrm{ng} / \mathrm{mL}$. Três pacientes $(10,7 \%)$ apresentaram níveis menores que $3 \mathrm{ng} / \mathrm{mL}$. Em oito pacientes $(28,6 \%)$ os níveis variaram entre 3 e $50 \mathrm{ng} / \mathrm{mL}$. Em sete pacientes (25\%) os níveis foram entre 50 e $100 \mathrm{ng} / \mathrm{mL}$. Em oito pacientes os níveis estiveram entre 100 e 150 $\mathrm{ng} / \mathrm{mL}$ e em dois $(7,1 \%)$, entre 150 e $200 \mathrm{ng} / \mathrm{mL}$

O número de metástases hepáticas variou de um a quatro. Em 19 pacientes $(67,8 \%)$ era única, em $7(25 \%)$ eram $2 ; 1$ paciente $(3,6 \%)$ apresentou 4 metástases.

\section{Ressecções hepáticas}

A distribuição das ressecções hepáticas é mostrada na Tabela 1. Foram realizadas 38 ressecções hepáticas em 28 pacientes, assim distribuídas: 3 ressecções em cunha (não-anatômicas), 16 segmentectomias, 7 lobectomias esquerdas, 8 hepatectomias direitas, 1 hepatectomia esquerda e 3 hepatectomias direitas ampliadas.

TABELA 1 - Ressecções hepáticas em 28 pacientes

\begin{tabular}{lr}
\hline Tipo de ressecção & $\mathbf{n}$ \\
\hline Ressecção em cunha & 3 \\
Segmentectomia & 16 \\
Lobectomia esquerda & 7 \\
Hepatectomia direita & 8 \\
Hepatectomia esquerda & 1 \\
Hepatectomia direita ampliada & 3 \\
Total & 38 \\
\hline
\end{tabular}


Em oito pacientes, foram realizadas mais de uma ressecção no fígado no mesmo tempo cirúrgico. Em 20 pacientes a ressecção foi única.

Três pacientes com recidiva de suas metástases no fígado surgidas no decorrer do seguimento foram levados à laparotomia com o intuito de ressecá-las. Um deles não pôde ser submetido a ressecção por apresentar metástase extra-hepática no diafragma nãoressecável. Os outros dois pacientes foram submetidos a nova ressecção hepática.

As margens foram consideradas livres de tumor quando superiores a $1 \mathrm{~cm}$ em todos os quadrantes. Assim, em 22 pacientes $(78,57 \%)$ foram obtidas margens livres de tumor com a ressecção, e em 6 $(21,43 \%)$ as margens não foram livres de tumor.

Em seis pacientes $(21,42 \%)$ submetidos a ressecção hepática, havia doença metastática extra-hepática, que foi completamente ressecada simultaneamente à ressecção da metástase hepática. Em cinco desses pacientes, a doença metastática extra-hepática estava localizada no interior da cavidade abdominal. Em um paciente, a doença metastática extra-hepática estava localizada no lobo inferior do pulmão direito, tendo sido ressecada através de segmentectomia pulmonar na mesma ocasião da ressecção hepática. Todas as metástases extra-hepáticas foram descobertas no transoperatório da ressecção hepática, exceto a pulmonar.

\section{Resultados pós-operatórios}

O tempo médio de internação foi de 11,32 dias. A mortalidade operatória (óbitos ocorridos até o $30^{0}$ dia de pós-operatório) foi de 3,6\% (um paciente, $5^{0}$ dia do período pós-operatório, por falência hepática aguda). Até a data do último seguimento (30 de setembro de 2001) de um total de 28 pacientes, 10 foram a óbito.

A morbidade foi de 39,3\% (11 pacientes com 15 complicações). As complicações pós-operatórias das ressecções hepáticas estão apresentadas na Tabela 2 .

TABELA 2 - Complicações pós-operatórias em 28 pacientes

\begin{tabular}{lc}
\hline Complicações pós-operatórias & n \\
\hline Fístula biliar & 4 \\
Ascite & 3 \\
Infecção da ferida operatória & 2 \\
Gastroparesia & 2 \\
Abcesso subfrênico & 1 \\
Infecção respiratória & 1 \\
Insuficiência cardíaca & 1 \\
Insuficiência hepática aguda & 1 \\
Total & 15 \\
\hline
\end{tabular}

\section{Sobrevida}

A curva de sobrevida de Kaplan-Meier (Figura 1) ilustra a sobrevida acumulada após as ressecções hepáticas. O tempo médio de seguimento dos pacientes foi de 3,7 anos, variando entre 5 dias a 9,5 anos. A sobrevida dos pacientes em 1 ano foi de 92,3\%, em 2, de $87 \%$, em 3 , de $77,3 \%$, em 4 , de $50 \%$, e em 5 , de $35 \%$.

A Tabela 3 demonstra um resumo geral dos resultados operatórios quanto ao número de pacientes, tempo de internação, mortalidade operatória, morbidade e tempo de sobrevida em 1, 2, 3, 4 e 5 anos.

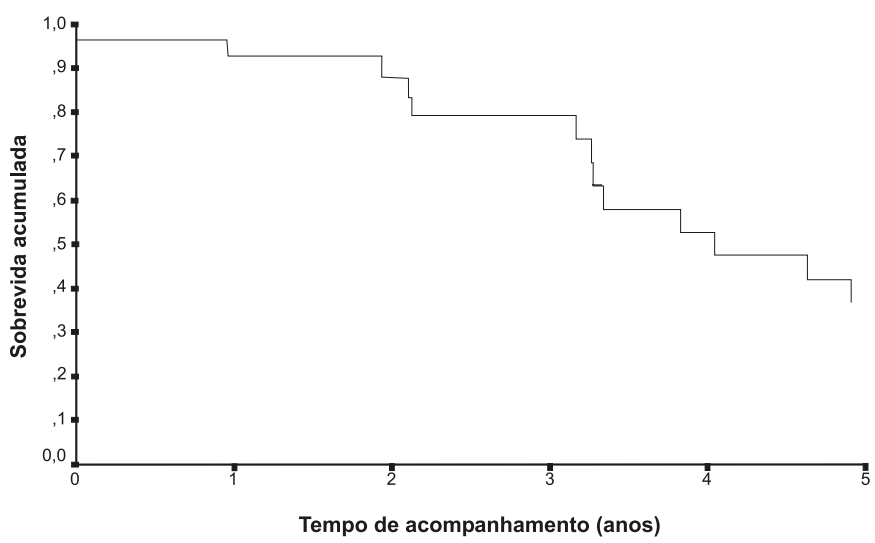

FIGURA 1 - Curva de Kaplan-Meier de sobrevida acumulada

TABELA 3 - Resumo dos resultados operatórios em 28 pacientes

\begin{tabular}{lc}
\hline Número de ressecções & 38 \\
\hline Tempo médio de internação (dias) & $11,3(5$ a 24$)$ \\
Mortalidade operatória (até o $\left.30^{\underline{0}} \mathrm{PO}\right)$ & $1(3,6 \%)$ \\
Sobrevida em 1 ano & $92,3 \%$ \\
Sobrevida em 2 anos & $87 \%$ \\
Sobrevida em 3 anos & $77,3 \%$ \\
Sobrevida em 4 anos & $50 \%$ \\
Sobrevida em 5 anos & $35 \%$ \\
\hline
\end{tabular}

\section{Análise estatística univariada dos fatores prognósticos em estudo}

1. Estágio Dukes C do tumor primário - os pacientes com tumor primário estágio Dukes C tiveram mortalidade significativamente maior no período de seguimento médio de 3,7 anos $(P=0,04)$, com probabilidade de morte 4,9 vezes maior, 
quando comparados com os pacientes com tumor primário estágio Dukes B.

2. Tamanho da metástase hepática maior que $5 \mathrm{~cm}-$ os pacientes com metástases hepáticas maiores do que $5 \mathrm{~cm}$ tiveram mortalidade significativamente maior no período médio de seguimento de 3,7 anos ( $P=0,04)$, com probabilidade de morte 3,1 vezes maior, quando comparados com os pacientes com metástases hepáticas menores do que $5 \mathrm{~cm}$.

3. Intervalo menor que 1 ano entre a ressecção do tumor primário e o surgimento da metástase hepática - os pacientes nos quais o intervalo de tempo entre a ressecção do tumor primário e o surgimento da metástase hepática foi menor do que 1 ano tiveram significativamente maior mortalidade no período médio de seguimento de 3,7 anos $(P=0,01)$, com probabilidade de morte 16 vezes maior, quando comparados com os pacientes nos quais o intervalo de tempo entre a cirurgia do tumor primário e o surgimento da metástase foi maior do que 1 ano.

4. Nível de CEA (antígeno carcinoembriônico) maior que $100 \mathrm{ng} / \mathrm{mL}$ - os pacientes com nível de CEA maior do que $100 \mathrm{ng} / \mathrm{mL}$ na avaliação diagnóstica inicial da metástase hepática tiveram significativamente maior mortalidade no período médio de seguimento de 3,7 anos $(P=0,02)$, com probalidade de morte 9,7 vezes maior, quando comparados com os pacientes com nível de CEA menor do que $100 \mathrm{ng} / \mathrm{mL}$ na avaliação diagnóstica inicial da metástase hepática.

5. Margens cirúrgicas menores que $1 \mathrm{~cm}$ - foi um fator que não obteve significância estatística $(P=0,28)$ como fator preditivo de mortalidade no período médio de seguimento de 3,7 anos; porém a probabilidade de morte nesse mesmo período foi 2 vezes maior quando as margens foram menores do que $1 \mathrm{~cm}$.
6. Presença de doença metastática extra-hepática - os pacientes com doença metastática extra-hepática concomitante com a metástase hepática (mesmo essa tendo sido ressecada com margens livres no mesmo tempo cirúrgico), tiveram significativamente maior mortalidade no período médio de seguimento de 3,7 anos $(P=0,04)$, com probabilidade de morte 7,3 vezes maior do que os pacientes sem doença metastática extra-hepática.

\section{Análise estatística multivariada dos fatores prognósticos em estudo}

$\mathrm{Na}$ análise multivariada apresentada na Tabela 4 , a presença de doença metastática extra-hepática foi significativa $(P=0,004)$ com probabilidade de morte 57,28 vezes maior. $O$ intervalo de surgimento da metástase menor do que 1 ano também foi significativo $(P=0,04)$, com probabilidade de morte 11,56 vezes maior.

O nível de CEA maior do que $100 \mathrm{ng} / \mathrm{mL}$ não foi significativo $(P=0,08)$, porém a probabilidade de morte foi 9,02 vezes maior.

O tamanho da lesão maior do que $5 \mathrm{~cm}$ não foi significativo $(P=0,12)$, porém a probabilidade de morte foi 3,3 vezes maior.

O estágio Dukes $\mathrm{C}$ do tumor primário não foi significativo $(P=0,12)$, porém a probabilidade de morte foi 4,3 vezes maior.

As margens cirúrgicas menores do que $1 \mathrm{~cm}$ não foram significativas, porém a probabilidade de morte foi 2 vezes maior.

Entre os fatores prognósticos estudados, a presença de doença metastática extra-hepática e um intervalo de surgimento da metástase menor do que 1 ano foram fatores prognósticos independentes de resultado adverso.

TABELA 4 - Análise multivariada dos fatores prognósticos em estudo

\begin{tabular}{lcrrr}
\hline & & & \multicolumn{2}{c}{$\mathbf{9 5 \%}$ IC para Exp ( B )\# } \\
\cline { 3 - 4 } & Significância $(\boldsymbol{P})$ & Exp (B)* & Inferior & Superior \\
\hline Margens $<1 \mathrm{~cm}$ & 0,420 & 1,990 & 0,374 & 10,578 \\
Doença extra-hepática + & 0,004 & 57,287 & 3,710 & 884,696 \\
Tamanho da metástase $>5 \mathrm{~cm}$ & 0,116 & 3,357 & 0,740 & 15,219 \\
Intervalo $<1$ ano & 0,047 & 11,564 & 1,028 & 130,032 \\
Tumor primário Dukes C & 0,120 & 4,314 & 0,684 & 27,223 \\
CEA $>100$ ng/mL & 0,082 & 9,024 & 0,754 & 108,056 \\
\hline
\end{tabular}

*Risco relativo ou hazard, ou probabilidade de morte

\# Intervalo de confiança de $95 \%$ para o risco relativo ou hazard ou probabilidade de morte 


\section{DISCUSSÃO}

As metástases hepáticas são a causa mais comum de morte em pacientes com câncer colorretal. A sobrevida de pacientes com metástases hepáticas não ressecadas varia de 5 a 19 meses, com média inferior a 9 meses $^{(29)}$. A quimioterapia isoladamente pode proporcionar sobrevida média de 15 meses ${ }^{(27,38)}$. A experiência de SCHEELE et al. ${ }^{(35)}$, em 434 ressecções de metástases hepáticas de câncer colorretal em período de 32 anos (de 1960 a 1992), mostrou sobrevida de 32\% em 10 anos e de $18 \%$ em 20 , com mortalidade operatória inferior a $5 \%$. A comparação entre esses métodos, evidenciando a clara superioridade do método cirúrgico para o tratamento das metástases hepáticas de câncer colorretal, tornou a ressecção hepática o tratamento padrão para metástases de câncer colorretal no fígado.

A ressecção hepática é um método terapêutico seguro e eficiente para as metástases hepáticas de câncer colorretal, constituindo-se no único tratamento que oferece a oportunidade de cura da doença ${ }^{(14)}$.

A análise de fatores prognósticos é utilizada para selecionar pacientes com vistas à ressecção hepática, delinear grupos de risco que necessitem terapia adjuvante pós-ressecção, fazer estimativa do tempo de sobrevida e oferecer aos pacientes dados objetivos sobre o seu prognóstico ${ }^{(39)}$.

Grande número de fatores de risco (clínicos e patológicos), que influem na sobrevida desses pacientes, tem sido relatados; porém, a seleção de pacientes a serem submetidos a ressecção hepática permanece controversa ${ }^{(16)}$. Tentativas de selecionar pacientes utilizando escore prognóstico foram propostas por NORDLINGER et al. ${ }^{(30)}$, em estudo baseado em 1.568 pacientes submetidos a ressecção de metástase hepática de câncer colorretal, por FONG et al. ${ }^{(14)}$, que analisaram 1.001 pacientes e por IWATSUKI et al. ${ }^{(21)}$, analisando 305 pacientes.

O objetivo deste trabalho foi determinar o impacto dos seguintes fatores prognósticos na sobrevida de pacientes submetidos a ressecção de metástases hepáticas de câncer colorretal: 1) estágio Dukes C do tumor primário; 2) tamanho da(s) metástase(s) maior que $5 \mathrm{~cm} ; 3$ ) intervalo menor que 1 ano entre a ressecção do tumor primário e o surgimento da metástase; 4) nível de CEA maior do que $100 \mathrm{ng} / \mathrm{mL}$; 5) margens livres de tumores menores do que $1 \mathrm{~cm}$ na metástase ressecada; 6) presença de doença metastática extra-hepática concomitante com a metástase hepática por ocasião da ressecção.

$\mathrm{O}$ estágio Dukes $\mathrm{C}$ do tumor primário no presente estudo foi estatisticamente significativo na análise univariada, com probabilidade de morte 4,9 vezes maior; porém, na análise multivariada, não foi estatisticamente significativo, mas a probabilidade de morte se manteve elevada (4,3 vezes maior). Resultados semelhantes foram observados por HARMS et al. ${ }^{(18)}$ em 449 pacientes. Outros autores encontraram significância na análise uni e multivariada e consideram o estágio Dukes C como fator prognóstico independente adverso à sobrevida, não se constituindo, porém, em contra-indicação para a ressecção hepática ${ }^{(7,14,19,20,22,30,35,40)}$.

O tamanho da metástase maior do que $5 \mathrm{~cm}$ na presente série foi estatisticamente significativo na análise univariada, com probabilidade de morte 3,1 vezes maior; na análise multivariada não foi significativo, mas a probabilidade de morte manteve-se elevada (3,3 vezes maior). Resultados semelhantes foram observados por IWATSUKI et al. ${ }^{(21)}$, em 305 pacientes submetidos a ressecção hepática de metástases colorretais, e WANG et al. ${ }^{(42)}$, em 54 pacientes. NORDLINGER et al. ${ }^{(30)}$, analisando 1.568 pacientes, observaram que o tamanho da metástase maior do que $5 \mathrm{~cm}$ foi fator prognóstico independente. Resultados semelhantes aos desses autores também foram observados por FONG et al. ${ }^{(14)}$, analisando 1.001 pacientes consecutivos submetidos a ressecção hepática de metástases colorretais. Por outro lado, JAECK et al. ${ }^{(22)}$ não encontraram significância uni ou multivariada em 1.818 pacientes.

Apesar dos dados controversos e conflitantes em relação a esse fator prognóstico e a todos os outros, há consenso na literatura ${ }^{(3,12,23}$ 26, 29,33,36,39,41,45): não há contra-indicação para a ressecção de metástase com tamanho maior do que $5 \mathrm{~cm}$.

O intervalo menor que 1 ano entre a ressecção do tumor primário e o surgimento da metástase nesta série obteve significância estatística na análise univariada, com probabilidade de morte 16 vezes maior, e também na análise multivariada, com probabilidade de morte 12 vezes maior. Foi, portanto, fator prognóstico adverso independente. Resultados semelhantes foram observados por FONG et al. ${ }^{(14)}$, estudando 1.001 pacientes consecutivos, por NORDLINGER et al. ${ }^{(30)}$ e GAYOWSKI et al. ${ }^{(16)}$. Outros estudos ${ }^{(33)}$ também consideram o intervalo de tempo entre a ressecção do tumor primário e o surgimento da metástase hepática fator prognóstico adverso independente. Resultados diferentes foram relatados por JAECK et al. ${ }^{(22)}$, CADY et al. ${ }^{(8)}$ e AMBIRU et al..$^{(3)}$, que não consideraram o intervalo de tempo entre a ressecção do tumor primário e o surgimento da metástase hepática como fatores prognósticos independentes. Apesar dos resultados discordantes a respeito da importância desse fator, há consenso na literatura ${ }^{(3,12,23,26,28,29,36,39,41,45)}$ de que o intervalo entre a ressecção do tumor primário e o surgimento da metástase no fígado não contra-indica a ressecção hepática.

O nível de CEA maior do que $100 \mathrm{ng} / \mathrm{mL}$ neste estudo, teve significância estatística na análise univariada, com probabilidade de morte 9,7 vezes maior, mas na análise multivariada não alcançou significância estatística, embora a probabilidade de morte tenha se mantido elevada ( 9 vezes maior). Resultados semelhantes foram observados por SUGAWARA et al. ${ }^{(41)}$, em 304 pacientes, e por LISE et al. ${ }^{(26)}$, em 135 pacientes. Alguns estudos têm demonstrado que o nível pré-operatório de CEA com pontos de corte variando entre 20 e $200 \mathrm{ng} / \mathrm{mL}$ é fator prognóstico independente ${ }^{(7,13,14,22,30,35,45)}$. Apesar de não haver unanimidade quanto a esse ser fator prognóstico 
independente, há consenso na literatura de que o nível pré-operatório de CEA, qualquer que seja, não contra-indica a ressecção hepática de metástases colorretais.

O fator prognóstico margens livres de tumor menores do que 1 $\mathrm{cm}$, no presente estudo, não apresentou significância estatística tanto na análise univariada, como na análise multivariada; a probabilidade de morte, porém, foi 2 vezes maior. Resultados semelhantes foram observados por DOCI et al. ${ }^{(12)}$, em 219 pacientes. SCHEELE et al. ${ }^{(35)}$ observaram, em 350 pacientes, diferença estatística pequena na sobrevida em 5 anos entre pacientes com margens de 1 até $9 \mathrm{~mm}$ (37\%) e pacientes com margens acima de 9 mm (43\%). JAMISON et al. ${ }^{(23)}$ observaram, em 280 pacientes, sobrevida em 5 anos de $17 \%$ quando a margem foi $0,29 \%$ quando foi de 0 a $1 \mathrm{~mm}, 30 \%$ quando foi de 2 a $10 \mathrm{~mm}$ e, finalmente, $36 \%$ quando foi superior a $1 \mathrm{~cm}$. Outros autores também consideram a margem inferior a $1 \mathrm{~cm}$ como fator prognóstico adverso independente ${ }^{(7,8,14,16,21,22,30)}$. Mesmo não sendo possível obter margem superior a $1 \mathrm{~cm}$, há consenso na literatura de que a metástase deva ser ressecada e todo o esforço cirúrgico deve ser feito para assegurar uma borda completa de tecido hepático não afetado macroscopicamente por tumor ${ }^{(36)}$.

A presença de doença metastática extra-hepática concomitante com a metástase hepática apresentou significância estatística na análise univariada, com probabilidade de morte 7,3 vezes maior e na análise multivariada 57,3 vezes maior, constituindo-se no mais importante fator prognóstico adverso independente do presente trabalho. Resultados semelhantes foram obtidos por HUGHES et al. ${ }^{(20}$ ${ }^{33)}$, em 800 pacientes, FONG et al. ${ }^{(14)}$, em 1.001 pacientes; IWATSUKI et al. ${ }^{(21)}$, em 305 pacientes e JAMISON et al. ${ }^{(23)}$, em 280 pacientes. De acordo com FONG et al. ${ }^{(14)}$, a presença de doença tumoral extrahepática representada ou por implantes peritoniais, ou em estruturas adjacentes, foi o fator prognóstico adverso independente com maior influência na série deles. D'ALBUQUERQUE(11), em trabalho realizado em São Paulo, contra-indicou a ressecção hepática quando foram encontradas metástases extra-hepáticas concomitantes.

Embora a presença de doença tumoral extra-hepática reduza a sobrevida em 5 anos, a níveis não superiores a $20 \%{ }^{(20)}$, a invasão direta por tumor a estruturas adjacentes, implantes localizados e metástases pulmonares não constitui contra-indicação para a ressecção hepática, desde que também possam ser ressecadas inteiramente e com margens livres ${ }^{(28,36)}$.

\section{CONCLUSÕES}

Os fatores prognósticos independentes com influência adversa na sobrevida foram: o intervalo entre a ressecção do tumor primário e o surgimento da metástase hepática menor que 1 ano e a presença de doença metastática extra-hepática.

Chedid AD, Villwock MM, Chedid MF, Rohde L. Prognostic factors following liver resection for hepatic metastases from colorectal cancer. Arq Gastroenterol 2003;40(3):159-165

ABSTRACT - Aim - To determine the impact of prognostic factors on survival of patients with metastases from colorectal cancer that underwent liver resection. Methods - The records of 28 patients that underwent liver resection for metastases from colorectal cancer between April 1992 and September 2001 were retrospectively analyzed. Thirty-eight resections were performed (more than one resection in eight patients and two patients underwent reresections). The primary tumor was resected in all the patients. A screening protocol for liver metastases including clinical examinations every three months, ultrassonography and CEA level until 5 years of follow-up and after every 6 months, was applied. The prognostic factors analyzed regarding the impact on survival were: Dukes $C$ stage of primary tumor, size of metastasis $>5 \mathrm{~cm}$, a disease-free interval from primary tumor to metastasis $<1$ year, CEA level $>100 \mathrm{ng} / \mathrm{mL}$, resection margins $<1 \mathrm{~cm}$ and extrahepatic disease. The Kaplan-Meier curves, log rank and Cox regression were used for the statistical analysis. Results - Perioperative morbidity and mortality were 39.3\% and 3.6\%, respectively. The 5-year survival rate was $35 \%$. The independent prognostic factors were: disease-free interval from primary tumor to metastasis $<1$ year and extrahepatic disease. Conclusions - The liver resection for metastases from colorectal cancer is a safe procedure with more than 30\% 5-year survival. Disease-free interval from primary tumor to metastasis $<1$ year and extrahepatic disease were independent prognostic factors.

HEADINGS - Colorectal neoplasms. Liver neoplasms. Neoplasm metastasis. Prognosis.

\section{REFERÊNCIAS BIBLIOGRÁFICAS}

1. Adson MA, van Heerden JA, Adson MH, Wagner JS, Ilstrup DM. Resection of hepatic metastases from colorectal cancer. Arch Surg 1984;119:647-51.

2. Altman DG. Practical statistics for medical research. London: Chapman \& Hall; 1991. p. 365-95.

3. Ambiru S, Miyzaki M, Isono T, Ito H, Nakagawa K, Shimizu H, Kusashio K, Furuya S, Nakajima N. Hepatic resection for colorectal metastases: analysis of prognostic factors. Dis Colon Rectum 1999;42:632-9.

4. Bengmark S, Hafstrom L. The natural history of primary and secondary malignant tumors of liver: the prognosis for patients with hepatic metastases from colonic and rectal carcinoma by laparotomy. Cancer 1969;23:198-202.
5. Bismuth H, Chiche L. Surgical anatomy and anatomical surgery of the liver. In: Blumgart LH, editor. Surgery of the liver and biliary tract. $2^{\text {nd }}$ ed. Edinburgh Churchill Livingstone; 1994. v.1.

6. Brasil. Ministério da Saúde. Instituto Nacional do Câncer. COMPREV. Estatística INCA do câncer de intestino grosso no Brasil. Rio de Janeiro; 2001.

7. Cady B, Stone MD, McDermott WV, Jenkins RL, Bothe A, Lavin PT, Lovett EJ, Steele GD. Technical and biological factors in disease-free survival after hepatic resection for colorectal cancer metastases. Arch Surg 1992;127:561-8.

8. Cady B, Jenkins RL, Steele GD, Lewis WD, Stone MD, McDermott WV, Jessup JM, Bothe A, Lalor P, Lovett EJ, Lavin P, Linehan DC. Surgical margin in hepatic resection for colorectal metastasis: a critical and improvable determinant of outcome. Ann Surg 1998,227:566-71. 
9. Couinaud C. Structures portal segmentation. In: Couinaud C. Controlled hepatectomies and exposure of the intrahepatic bile ducts: an anatomical and technical study. Paris: Imprimeries Réunies de Chambéry; 1981.

10. Couinaud C. Structures terminology of the main principles of the hepatic surgery. In: Couinaud C. Controlled hepatectomies and exposure of the intrahepatic bile ducts. Paris: Imprimeries Réunies de Chambéry; 1981.

11. D'Albuquerque LAC. Hepatectomias no tratamento das metástases hepáticas de câncer colorretal [tese]. São Paulo: Faculdade de Medicina da Universidade de São Paulo; 1997

12. Doci R, Bignami P, Montalto F, Gennari L. Prognostic factors for survival and disease -free survival in hepatic metastases from colorectal cancer treated by resection. Tumori 1995;81 Suppl:143-6.

13. Fong Y, Cohen AM, Fortner JG, Enker WE, Turnbull AD, Coit DG, Marrero AM Prasad M, Blumgart LH, Brennan MF. Liver resection for colorectal metastases. J Clin Oncol 1997;15:938-46.

14. Fong Y, Fortner J, Sun L, Brennan MF, Blumgart LH. Clinical score for predicting recurrence after hepatic resection for metastatic colorectal cancer. Analysis of 1001 consecutive cases. Ann Surg 1999;230:309-21.

15. Foster JH, Bermann MM. Solid liver tumor. In: Ebert PA, editor. Major problems in clinical surgery. Philadelphia: WB Saunders; 1977. p. 209-34.

16. Gayowski TJ, Iwatsuki S, Madariaga JR, Selby R, Todo S, Irish W, Starzl TE. Experience in hepatic resection for metastatic colorectal cancer: analysis of clinical and pathologic risk factors. Surgery 1994;116:703-11.

17. Goldsmith NA, Woodburne RT. The surgical anatomy pertaining to liver resection. Surg Gynecol Obstet 1957;195:310-8.

18. Harms J, Obst T, Thorban S, Busch R, Fink U, Heidecke CD, Roder JD, Ssiewert JR. The role of surgery in the treatment of liver metastases for colorectal cancer patients. Hepatogastroenterology 1999;46:2321-8.

19. Hughes KS, Simon R, Songhorabodi S, Adson MA, Ilstrup DM, Fortner JG, Maclean BJ, Foster JH, Daly JM, Fitzherbert D. Resection of the liver for colorectal carcinoma metastases: a multi-institutional study of patterns of recurrence. Surgery 1986;100:278-84.

20. Hughes KS, Scheele J, Sugarbaker PH. Surgery for colorectal cancer metastatic to the liver optimizing the results of treatment. Surg Clin North Am 1989;69:33959.

21. Iwatsuki S, Dvorchik I, Madariaga JR, Marsh JW, Dodson F, Bonham AC, Geller DA, Gayowski TJ, Fung JJ, Starzl TE. Hepatic resection for metastatic colorecta adenocarcinoma: a proposal of a prognostic scoring system. J Am Coll Surg 1999;189:291-9.

22. Jaeck D, Bachellier P, Guiguet M, Boudjema K, Vaillant JC, Balladur P, Nordlinger B. Long-term survival following resection of colorectal hepatic metastases. Association Française de Chirurgie. Br Jr Surg 1997;84:977-80.

23. Jamison RL, Donohue JH, Nagorney DM, Rosen CB, Harmsen WS, Ilstrup DM. Hepatic resection for metastatic colorectal cancer results in cure for some patients. Arch Surg 1997;132:505-11

24. Jarnagin WR, Fong Y, Ky A, Schwartz LH, Paty PB, Cohen AM, Blumgart LH Liver resection for metastatic colorectal cancer: assessing the risk of occult irresectable disease. J Am Coll Surg 1999;188:33-42.

25. Lehnert T, Knaebel HP, Dück M, Bülzebruck H, Herfarth C. Sequential hepatic and pulmonary resections for metastatic colorectal cancer. Br J Surg 1999;86:2413.

26. Lise M, Bacchetti S, Da Pian P, Nitti D, Pilati P. Patterns of recurrence after resection of colorectal liver metastases: prediction by models of outcome analysis. World J Surg 2001;24:638-44
27. Lise M, Da Pian PP, Nitti D, Pilati PL. Colorectal metastases to the liver: present results and future strategies. J Surg Oncol 1991;Suppl 2:69-73.

28. Nagakura S, Shirai Y, Yamato Y, Yokoyama N, Suda T, Hatakeyama K Simultaneous detection of colorectal carcinoma liver and lung metastases does not warrant resection. J Am Coll Surg 2001;193:153-60.

29. Nagorney DM. Hepatic resections for metastases from colorectal cancer. Prob Gen Surg 1987;4:83-92.

30. Nordlinger B, Guiguet M, Vaillant JC, Balladur P, Boudjema K, Bachellier P, Jaeck D. Surgical resection of colorectal carcinoma metastases to the liver. A prognostic scoring system to improve case selection, based on 1568 patients Association Française de Chirurgie. Cancer 1996;77:1254-62.

31. Pietra N, Sarli L, Costi R, Ouchemi C, Grattarola M, Peracchia A. Role of followup in management of local recurrences of colorectal cancer: a prospective, randomized study. Dis Colon Rectum 1998;41:1127-33.

32. Ramming KP. Diseases of the colon and rectum. In: Sabiston DC, editor. Sabiston essentials of surgery. Philadelphia: WB Saunders; 1987. p. 490-1.

33. Resection of the liver for colorectal carcinoma metastases: a multi-institutional study of indications for resection. Registry of Hepatic Metastases. Surgery 1988; 103:277-88.

34. Rosen CB, Nagorney DM, Taswell HF, Helgeson SL, Ilstrup DM, van Heerden JA, Adson MA. Perioperative blood transfusion and determinants of surviva after liver resections for metastatic colorectal carcinoma. Ann Surg 1992;216:493504.

35. Scheele J, Altendorf-Hofmann A. Resection of colorectal liver metastases. Langenbecks Arch Surg 1999;384:313-27.

36. Scheele J, Stang1 R, Altendorf-Hofmann A, Paul M. Resection of colorectal liver metastases. World J Surg 1995; 19:59-71.

37. Schlag P, Hohengerger P, Herfarth C. Resection of liver metastases in colorecta cancer: competitive analysis of treatment results in synchronous versus metachronous metastases. Eur J Surg Oncol 1990;16:360-5.

38. Sheiner PA, Brower ST. Treatment of metastatic cancer to the liver. Semin Liver Dis 1994;14:169-77.

39. Smith JA, De Matteo RP, Fong Y. Metastatic liver disease. In: Blumgart LH Fong Y, Jarnagin WR, American Cancer Society, editors. Hepatobiliary cancer Hamilton, Ont.: B C Decker; 2001. p. 75-95.

40. Stang1 R, Altendorf-Hofmann A, Chanrley RM, Scheele J. Factors influencing the natural history of colorectal liver metastases. Lancet 1994;343:1405-10.

41. Sugawara Y, Yamamoto J, Yamasaki S, Shimada K, Kosuuge T, Makuuchi M. Estimating the prognosis of hepatic resection in patients with metastatic liver tumors from colorectal cancer with special concern for the timing of hepatectomy. Surgery 2001;129:408-13.

42. Wang JY, Chiang J-M, Jeng L-B, Changchien CR, Chen JS, Hsu KC. Resection of liver metastases from colorectal cancer: are there any truly significant clinical prognosticators? Dis Colon Rectum 1996;39:847-51.

43. Wilson SM, Adson MA. Surgical treatment of hepatic metastases from colorecta cancers. Arch Surg 1976;111:330-4

44. Woodington GF, Waugh JM. Results of resection of metastatic tumors of the liver. Am J Surg 1963;105:24-9.

45. Younes RN, Rogatko A, Brennan MF. The influence of intra-operative hypotension and peri-operative blood transfusion on disease free survival in patients with complete resection of colorectal liver metastases. Ann Surg 1991;214:107-13.

Recebido em 29/11/2002. Aprovado em 30/1/2003 